\title{
Cultural influence on the aesthetic judgment of architecture
}

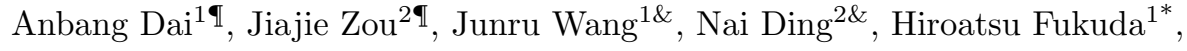 \\ 1 Department of Architecture, The University of Kitakyushu, Kitakyushu 808-0135, \\ Japan \\ 2 College of Biomedical Engineering and Instrument Sciences, Zhejiang University, \\ 310027 Hangzhou, China \\ * fukuda@kitakyu-u.ac.jp
}

\begin{abstract}
Architects should consider the aesthetic experience of potential users when designing architectures. Previous studies have shown that subjective aesthetic judgment of architectures is influenced by Structure features, and Western observers prefer structures that have curvilinear contours, high ceilings, and open spaces. The common building styles, however, vary across cultures, and may potentially influence observer's aesthetic preference. It remains unclear whether the preference for contours, ceiling height, and openness is universal or culture dependent. To investigate this issue, this study analyzes the aesthetic judgment of Chinese observers, and the results demonstrate that Chinese observers also prefer high ceilings and open spaces, similar to previous results based on Western observers. Preference for curvilinear contours is also observed but strongly interacts with ceiling height and openness. Post hoc analysis reveals that Chinese observers prefer curvilinear contours only when the ceiling is low and the space is closed. In sum, these results suggest that preferences for high ceilings and open spaces are observed in both Western cultures and in the Chinese culture. The preference for curvilinear contours, however, is less reliable in the Chinese culture.
\end{abstract}

\section{Introduction}

Investigating the preference of architectural features from the perspective of empirical aesthetics allows architects to gather more information about how to design structures that can meet both functional and public aesthetic requirements. Environmental characteristics can trigger neurological and physiological responses in humans, thereby exerting a positive or negative impact on them. To a certain extent, a good architectural design enhances users' comfort, cognition and creativity [1. Architectural aesthetics connects emotion and aesthetics and strikes a balance between the two [2].

Previous studies have demonstrated the reward circuitry in the brain is activated when seeing artwork. Artists who know how to exploit this circuitry can intensify an individual's aesthetic experience 3 . Once a certain architectural element fits in a certain life scene, such as work, study, and rest, it can enhance behavioral effects through positive emotions. Currently, many architects have such ideas but lack the theoretical foundation as well as an understanding of the effect of some architectural factors on subjective experience.

Studies have shown that the aesthetic judgment of architecture is influenced by several factors, including contour, ceiling height, and openness [4. A study conducted by researchers in Spain, Canada, and Denmark showed that Western observers prefer 
structures with curvilinear contours, high ceilings, and open spaces 5 . Furthermore, functional magnetic resonance imaging (MRI) results indicated that more individuals believe that curvilinear contours are more beautiful than rectilinear contours and that environments with curvilinear contours can affect users' behaviors, emotions, and brain function 5,8. Ceiling height and openness also impact people's perceptions and emotions in built environments [9,10]. In structures with high ceilings, people tend to have more positive emotional responses, such as "happiness", "comfort" and "fun". Similarly, they tend to experience more positive emotions in spacious environments than in small environments 11,12.

Although it has been demonstrated that contour, ceiling height, and openness are critical factors that influence the aesthetic judgment of architecture, it remains unclear whether these factors influence aesthetic judgment in similar ways across cultures. In other words, is the aesthetic judgment of architecture a nature versus nurture issue? It remains unclear whether the aesthetic judgment of architecture reflects human nature that is common across cultures or is influenced by observer experience. If preferences for architectural features is influenced by daily architectural aesthetic experience, the aesthetic judgment of architecture may vary across cultures because building styles vary across cultures 13, 14.

Here, we investigate the aesthetic preference of Chinese observers using the same set of pictures used in a previous study conducted by Western researchers 5]. Because East Asians and Westerners see and think about the world in very different ways. [15 We test whether the Chinese observers show similar contour, ceiling height, and openness preferences as Western observers tested in Spain.

\section{Methods}

\section{Subjects}

The subjects in this study were college students who were all right-handed, had no visual impairments and color blindness, had normal or corrected vision, and had no history of psychosis or neuropathy. The experimental protocol for this study was approved by the Research Ethics Committee of Zhejiang University School of Medicine (2019-047). Before the experiment, all participants signed a written informed consent form, and after completing the experiment, each participant received a reward. Candidate participants who had knowledge about the design and process of this experiment were excluded. Among 34 qualified subjects, 29 were recruited, including 19 males (age: $23.05 \pm 1.99$ years) and 10 females (age: $23.00 \pm 2.00$ years). Among the subjects, 9 were undergraduates, 13 were master's students, and 7 were PhD students.

Two hundred pictures of architectural spaces with different styles composed the stimulus material used this study. Each picture contained 3 factors, i.e., ceiling height, openness and contours, and each factor had 2 levels. Eight sets of pictures with different styles were generated to combine factors and factor levels, and each set contained 25 pictures, as shown in Fig 1. The pictures are from Ref [5].

The experiment was conducted in a sound-proof booth. The subject used 5 buttons (1 to 5) on the left-hand side for scoring and 1 button (0) on the right-hand side for starting the test; the buttons were attached with rubber tape for easy finger recognition. On the edge of the table, there was an adjustable chin rest to enable the subject to rest his/her chin snugly on the chin rest after sitting and to fix his/her line of sight to the central upper quarter of the display screen.

Before the experiment, the instructor guided the subject to sit correctly, put his/her hands on the corresponding buttons and relax; the subject could adjust the height of the seat so that his/her chin could rest snugly on the chin rest. During the experiment, 
the subject was asked to keep his/her posture steady, without moving his/her chin. The instructor then left the booth, and the subject pressed the 0 button to start the experiment.

The experiment included 200 trials, and 1 picture was presented in each trial. The steps for each trial were as follows: first, a fixation point appeared in the center of the screen, lingering for $1 \mathrm{~s}$, and then, a random stimulus picture was displayed for $3 \mathrm{~s}$, which was then followed by 2 questions that popped up on the screen, asking the subject to score the picture that was just displayed in terms of pleasantness and beauty (1=very unpleasant/ugly; $5=$ very pleasant/beautiful). The 200 trials were conducted in 4 sessions, and the subject rested after each session. The total duration of the experiment lasted approximately 35-75 minutes.

The experimental program was written using the MATLAB 2018 psychtoolbox software (ref).

Fig 1. Stimulus. A total of 200 pictures were divided into 8 groups of 25 pictures each. Each picture contained 3 notable aspects: ceiling height, degree of openness and contour type.

\section{Data analysis}

The experiment adopted a three-way repeated measures design, in which the 3 factors were ceiling height, openness and contour, which were all intra-group factors.

Specifically, to examine the influence of ceiling height, openness and contour on the viewer's perceived pleasantness and beauty, as well as the possible interaction effect, three-way intra-group repeated measures analysis of variance (ANOVA) was adopted. First, for each subject, the sum of the scores for the 25 pictures in each set was calculated and treated as one "repeated measures" result for that subject (with a value range of 25-125 points). Because each subject was tested using 8 sets of pictures, 8 "repeated measures" results were obtained for each subject. Finally, the scoring results for all subjects were used as response variables, and the 3 factors, i.e., ceiling height, openness, and contour, were used as intra-group factors in the repeated measures ANOVA model.

The three-way repeated measures ANOVA model included 3 main effect terms (ceiling height, openness, and contour), three two-way interaction terms (ceiling height $\times$ openness, openness $\times$ contour, and ceiling height $\times$ contour $)$ and one three-way interaction term (ceiling height $\times$ openness $\times$ contour). First, the total variation was decomposed to set up an ANOVA table based on model structure, and then, the significance of the main effect and if the interaction effects of each factor was tested. If a three-way interaction item was statistically significant, then a simple-simple effect test was performed, i.e., under different experimental treatments of the combination of 2 factors, the influence of the remaining factor on the dependent variable was tested. In addition, because the simple-simple effect analysis in this study was part of the overall ANOVA, it was unnecessary to make any additional adjustment to the p-value. (Notably, simple-simple effects per se do NOT need any form of p-value adjustment because simple effect analyses are still "omnibus F-tests").

All the data in this study were analyzed using the bruceR and tidyverse packages of $\mathrm{R}$ (version 3.6.3), and two-sided tests were performed, for which the significance level was set to $=0.05$. 


\section{Results}

The participants separately rated the pleasantness and beauty of each architectural space after viewing it for $3 \mathrm{~s}$. We first analyzed the beauty rating using 3-way repeated measures ANOVA (ceiling height $\times$ openness $\times$ contour). The 3 main factors significantly influenced the beauty rating Table 1. The ratings are shown in Fig 2 in general, architectural spaces with higher ceilings were rated as more beautiful than architectural spaces with lower ceilings. Architectural spaces that featured open space were rated as more beautiful than architectural spaces with less open space.

Furthermore, architectural spaces with curvilinear contours were rated as more beautiful than architectural spaces with rectilinear contours.

There was also a significant 3-way interaction between ceiling height, openness and contour. Simple-simple effect tests revealed that the observers always preferred higher ceilings and open spaces Fig 3 . However, ceiling height and openness modulated how contour influences the beauty rating Fig 3 . For architectural spaces with lower ceilings and less open space, curvilinear contours were rated higher than rectilinear contours $(\mathrm{p}<0.05)$.

In the combination of lower ceilings and more open space, rectilinear contours were more likely to lead to a high beauty score $(\mathrm{p}<0.05)$. When buildings have lower ceilings and rectilinear contours or higher ceilings and rectilinear contours or higher ceilings and curvilinear contours, more open space is more likely to lead to a higher beauty score than is less open space $(\mathrm{p}<0.05)$. Among the 4 combinations, compared to lower ceilings, higher ceilings were more likely to lead to a higher beauty score $(\mathrm{p}<0.05)$. The main effects of ceiling height and openness were statistically significant Table 2 , The ratings are shown in Fig 4 in general, comparted to those showing lower ceilings or less open spaces, pictures showing higher ceilings or open spaces were more likely to lead to a higher pleasantness scores; however, there was no significant difference in the pleasantness score between pictures showing curvilinear contours and those showing rectilinear contours.

We then analyzed the pleasantness rating using 3-way repeated measures ANOVA (ceiling height $\times$ openness $\times$ contour). The pleasantness rating was influenced by 2 of the 3 main factors, i.e., ceiling height and openness Table 2 .

Fig 5 shows that under different combinations of ceiling height and openness, the main effect of contour varied. For enclosed buildings with lower ceilings and for open buildings with higher ceilings, curvilinear contours, compared to rectilinear contours, were more likely to lead to higher pleasantness scores $(\mathrm{p}<0.05)$. For building with lower ceilings and more open space, rectilinear contours were more likely to lead to higher pleasantness scores $(\mathrm{p}<0.05)$. For different combinations of ceiling height and contour, the main effect of openness varied. For buildings with lower ceilings and rectilinear contours, higher ceilings and rectilinear contours, or higher ceilings and curvilinear contours, open space, compared to enclosed space, was more likely to lead to higher pleasantness scores $(\mathrm{p}<0.05)$. For different combinations of contour and openness, the main effect of ceiling height varied. Only for buildings that were enclosed and had rectilinear contours or that were open and had curvilinear contours did ceiling height have a statistically significant effect on pleasantness scores $(\mathrm{p}<0.05)$.

Fig 2. Scores of Beauty. Shows the aesthetic value score given by each group in experiment. The first digit of the abscissa represents the ceiling height, the second digit represents the degree of openness, and the third digit represents the contour type. 
Fig 3. Beauty Rating in Different Conditions. The influence of three building factors on the beauty value score in experiment. A shows the main effect of contour type with different combinations of ceiling height and degree of openness. B shows the main effect of the degree of openness with different combinations of ceiling height and contour type. C shows the main effect of ceiling height with different combinations of contour type and degree of openness.

Fig 4. Scores of Pleasantness. Shows the pleasure value score given by each group in experiment. The first digit of the abscissa represents the ceiling height, the second digit represents the degree of openness, and the third digit represents the contour type.

Fig 5. Pleasantness Rating in Different Conditions. The influence of three building factors on the beauty value score in experiment. A shows the main effect of contour type with different combinations of ceiling height and degree of openness. B shows the main effect of the degree of openness with different combinations of ceiling height and contour type. $\mathrm{C}$ shows the main effect of ceiling height with different combinations of contour type and degree of openness.

Table 1. ANOVA table for pleasantness scores .

\begin{tabular}{lllllllll}
\hline Factors & MS & MSE & df1 & df2 & F & p & $\eta^{\mathbf{2} p}$ & $\eta^{\mathbf{2} p 90 \% C I}$ \\
\hline Ceilingheight & 2278.142 & 30.357 & 1 & 28 & 75.05 & $<0.001^{*}$ & 0.728 & $0.554 \sim 0.805$ \\
\hline Degreeofopenness & 2793.211 & 29.622 & 1 & 28 & 94.3 & $<0.001^{*}$ & 0.771 & $0.618 \sim 0.835$ \\
\hline Contourtype & 157.246 & 14.889 & 1 & 28 & 10.56 & 0.003 & 0.274 & $0.064 \sim 0.456$ \\
\hline Ceilingheight $\times$ Degreeofopenness & 10.349 & 8.581 & 1 & 28 & 1.21 & 0.281 & 0.041 & $0.000 \sim 0.201$ \\
\hline Ceilingheight $\times$ Contourtype & 29.694 & 9.158 & 1 & 28 & 3.24 & 0.083 & 0.104 & $0.000 \sim 0.287$ \\
\hline Degreeofopenness $\times$ Contourtype & 422.28 & 11.298 & 1 & 28 & 37.38 & $<0.001^{*}$ & 0.572 & $0.342 \sim 0.691$ \\
\hline Ceilingheight $\times$ Degreeofopenness $\times$ Contourtype & 466.556 & 14.681 & 1 & 28 & 31.78 & $<0.001^{*}$ & 0.532 & $0.295 \sim 0.661$ \\
\hline
\end{tabular}

${ }^{*} \mathrm{p}<0.05$ Some scholars have suggested that the evaluation criteria of $\eta^{2} \mathrm{p}$ are small effect $(\geq 0.01$ and $<0.06)$, medium effect $(\geq$ 0.06 and $<0.14)$, and large effect $(\geq 0.14)$, but there is no unified evaluation standard at present. Usually, the effect is reported as $90 \%$ CI rather than $95 \%$ CI.

Table 2. ANOVA table for aesthetics scores .

\begin{tabular}{lllllllll}
\hline Factors & MS & MSE & df1 & df2 & F & p & $\eta^{\mathbf{2}} p$ & $\eta^{\mathbf{2} p 90 \% C I}$ \\
\hline Ceilingheight & 1630.28 & 30.539 & 1 & 28 & 53.38 & $<0.001^{*}$ & 0.656 & $0.451 \sim 0.753$ \\
\hline Degreeofopenness & 3150.97 & 28.907 & 1 & 28 & 109 & $<0.001^{*}$ & 0.796 & $0.656 \sim 0.853$ \\
\hline Contourtype & 29.694 & 12.792 & 1 & 28 & 2.32 & 0.139 & 0.077 & $0.000 \sim 0.253$ \\
\hline Ceilingheight $\times$ Degreeofopenness & 0.108 & 10.706 & 1 & 28 & 0.01 & 0.921 & 0.000 & $0.000 \sim 0.030$ \\
\hline Ceilingheight $\times$ Contourtype & 0.349 & 13.358 & 1 & 28 & 0.03 & 0.873 & 0.001 & $0.000 \sim 0.059$ \\
\hline Degreeofopenness $\times$ Contourtype & 390.522 & 17.173 & 1 & 28 & 22.74 & $<0.001^{*}$ & 0.448 & $0.205 \sim 0.598$ \\
\hline Ceilingheight $\times$ Degreeofopenness $\times$ Contourtype & 800.694 & 12.614 & 1 & 28 & 63.48 & $<0.001^{*}$ & 0.694 & $0.504 \sim 0.78$ \\
\hline
\end{tabular}

${ }^{*} \mathrm{p}<0.05$ Some scholars have suggested that the evaluation criteria of $\eta^{2} \mathrm{p}$ are small effect $(\geq 0.01$ and $<0.06)$, medium effect $(\geq$ 0.06 and $<0.14)$, and large effect $(\geq 0.14)$, but there is no unified evaluation standard at present. Usually, the effect is reported as $90 \%$ CI rather than $95 \%$ CI.

\section{Discussion}

The current experiment showed that buildings with higher ceilings elicit the sense of beauty and pleasantness more than buildings with lower ceilings. Ceiling height significantly modulated both the beauty rating and the pleasantness rating. Studies have also suggested different ceiling height for architectures of different functions. For 
example, rooms with lower ceilings may be more suitable for children to play quiet games, while those with higher ceilings are more suitable for them to play emotionally active games 16 . Notably, in rooms with a large area, the impact of ceiling height is more profound [17]. For example, some scholars suggest that the ceiling height of a conference room or hotel lobby with a larger area should be higher than $3.65 \mathrm{~m}$, so as to minimize the possibility of people feeling oppressed [18.

Openness also significantly modulated both beauty ratings and pleasantness ratings. Compared to those with enclosed spaces, buildings with open spaces always led to higher beauty and pleasant ratings, except for the condition with lower ceilings and curvilinear contours. The perceived openness can be viewed as the perceived mobility level in a space, which may affect people's aesthetic judgment of the space 11, 19,20. This study found that independent of ceiling height and contour combinations, open spaces had advantages over enclosed spaces in terms of pleasantness. Only for buildings with lower ceilings and curvilinear contours did openness not show a significant advantage, likely because low ceilings and curved contours in open spaces do not have substantial visual effects.

Contour had a less reliable influence on aesthetic judgment. It significantly influenced beauty ratings but not pleasantness ratings. Simple-simple effect analyses revealed that the main effect of contour was not significant, indicating that overall, the contour cannot independently trigger viewers' perceived pleasantness. For buildings that were enclosed and had lower ceilings, curvilinear contours tended to lead to higher beauty ratings and higher pleasantness ratings. This finding is consistent with the conclusion of many previous studies that people favor curvilinear contours more than rectilinear contours 7,9,21. However, for buildings that were open and had lower ceilings, this study found that rectilinear contours were more likely to trigger viewers' perceived pleasantness. When examining the effect of openness on perceived pleasantness under different combinations, independent od the combination of ceiling height and contour, open spaces always had an advantage over enclosed spaces in terms of triggering viewers' perceived pleasantness, except for buildings with lower ceilings and curvilinear contours, likely because low ceilings and curvilinear contours in open spaces have very little visual impact.

After comparing the results of this study with those of previous studies, we postulate that differences in viewers' perceptions, especially those regarding the aesthetic judgment of contours, is potentially related to cultural background. Eastern and Western aesthetics. Among the environmental burdens that may affect perception are the artistic products of a culture. There are clear differences between Eastern and Western art [15]. Literature on the differences between traditional Chinese and Western buildings can provide potential insights. In the Western culture, building design has focused on the individuality of each building, while in China, buildings are often organized into a hierarchal system [13. Traditional Chinese architecture tends to fragment outdoor spaces and integrate outdoor spaces with indoor spaces, and relatively simple indoor design guide people's attention to the outdoors. In contrast, buildings in the West, especially the public buildings, are renowned for the size and artistry of indoor spaces, and thus, guide people's attention to the center of squares $[22$. Furthermore, compared with Western architecture, Chinese architecture blends more with nature 23], i.e., the buildings are built in accordance with local conditions, such as terrain or surroundings, with many prominent representations all over China. Many factors might have lead to the differences between Chinese and Western architecture, e.g., history, society, technology, and religion. Chinese mainly used timber to build, while Westerners mainly used stone. The differences in materials lead to differences in building shapes and styles. Furthermore, in China, unified architectural styles have been promoted in the history $13,14,24$. 


\section{Conclusion}

211

this study suggested that the preference for high ceiling and open space is common in Chinese and Western cultures, while the preference for curvilinear contours is less reliable observed in the Chinese culture. Taken together, these results may provide architects with some insights when designing structures, allowing them to have a better understanding of the public's aesthetic preference.

\section{Supporting information}

S1 Fig. the correlation. the image of each group ceiling height with openness and contour in experimental test, and the correlation between the average score of pleasure and beauty of each subject.

\section{Conflict of Interest Statement}

The authors declare that the research was conducted in the absence of any commercial or financial relationships that could be construed as a potential conflict of interest.

\section{References}

1. Sternberg, E.M. and M.A. Wilson. Neuroscience and Architecture: Seeking Common Ground. Cell 2006. 127(2):p. 239-242

2. Arbib, M. and I. Lab. QA: In Pursuit of Brains for Buildings. 2016

3. Chatterjee, A. and O. Vartanian Neuroaesthetics. Trends in Cognitive Sciences 2014. 18(7): p. $370-375$.

4. Le Corbusier Vers une architecture. 1948.

5. Vartanian, O., et al. Impact of contour on aesthetic judgments and approach-avoidance decisions in architecture. Proceedings of the National Academy of Sciences 2013. 110(Supplement_2): p. 10446-10453.

6. Leder, H. and C. Carbon Dimensions in appreciation of car interior design. Applied Cognitive Psychology 2005. 19(5): p. 603-618.

7. Bar, M. and M. Neta Humans Prefer Curved Visual Objects. Psychological Science 2006. 17(8): p. 645-648.

8. Dazkir, S.S. and M.A. Read Furniture Forms and Their Influence on Our Emotional Responses Toward Interior Environments. Environment and Behavior 2011. 44(5): p. 722-732.

9. Franz, G., M. von der Heyde and H.H. Bülthoff An empirical approach to the experience of architectural space in virtual reality - exploring relations between features and affective appraisals of rectangular indoor spaces. Automation in Construction, 2005. 14(2): p. 165-172.

10. Meyers Levy, J., R.J. Zhu and D.S.A.E. John The Influence of Ceiling Height: The Effect of Priming on the Type of Processing That People Use Journal of Consumer Research, 2007. 34(2): p. 174-186. 
11. Stamps, A.E. Effects of Permeability on Perceived Enclosure and Spaciousness. Environment and Behavior 2010. 6(42): p. 864-886.

12. .III, A.E.S., Effects of Area Height,Elongation, and Color on Perceived Spaciousness. Environment and Behavior, 2011. 2(43): p. 252-273.

13. Liu, T On the differences between Chinese and Western traditional Architectural Culture. Time + Architecture 1989(03): p. 13-17.

14. Kang, Q Architecture, Space and Morphology — on the Architecture Morphology. JOURNAL OF SOUTHEAST UNIVERSITY ( Natural Science Edition) 2000. 30(1): p. 1-9.

15. Richard E. Nisbett, T.M. Culture and point of view. PNAS 2003. 19(100): p. 11163-11170.

16. G. Moore, C.L.A.H. Recommendations for Child Care Centers 1996: Cent. Archit. Urban Plan. Res. Books.

17. F. D. K. Ching, C.B Interior design illustrated 3rd ed. 2012: John Wiley Sons.

18. Seung Hyun Cha, C.K.T.W. Spatial perception of ceiling height and type variation in immersive virtual environments. Building and Environment 2019: p. 106285.1-106285.10.

19. Stamps, A.E. Visual permeability, locomotive permeability, safety, and enclosure. . Environment and Behavior 2005(37): p. 587-619.

20. Stamps, A.E.K.V. Perceived enclosure of space, angle above observer, and distance to boundary. Perceptual and Motor Skills 2004(99): p. 1187-1192.

21. Oshin Vartanian, et al. Architectural Design and the Brain: Effects of Ceiling Height and Perceived Enclosure on Beauty Judgments and Approach-avoidance Decisions. Journal of Environmental Psychology 2015. 41(3): p. 10-18.

22. Yu, Y. COMPARISON BETWEEN THE ORIENTAL AND WESTERN ARCHITECTURE Fujian Architecture Construction, 2001(4): p. 23-26.

23. Li, D. The differences of the eastern and weatern aesthetic psychology in the aesthetic atmosphere of the ancient eastern and western architecture. SCIENCE TECHNOLOGY INFORMATION 2008(34): p. 234.

24. Liu, T. Cultural tradition and Architecture- A brief discussion on the reasons for the differences between Chinese and Western Architecture. Quarterly Journal of the Shanghai Academy of Social Sciences 1986(01): p. 15-25. 
Fig 2. Scores of Beauty.

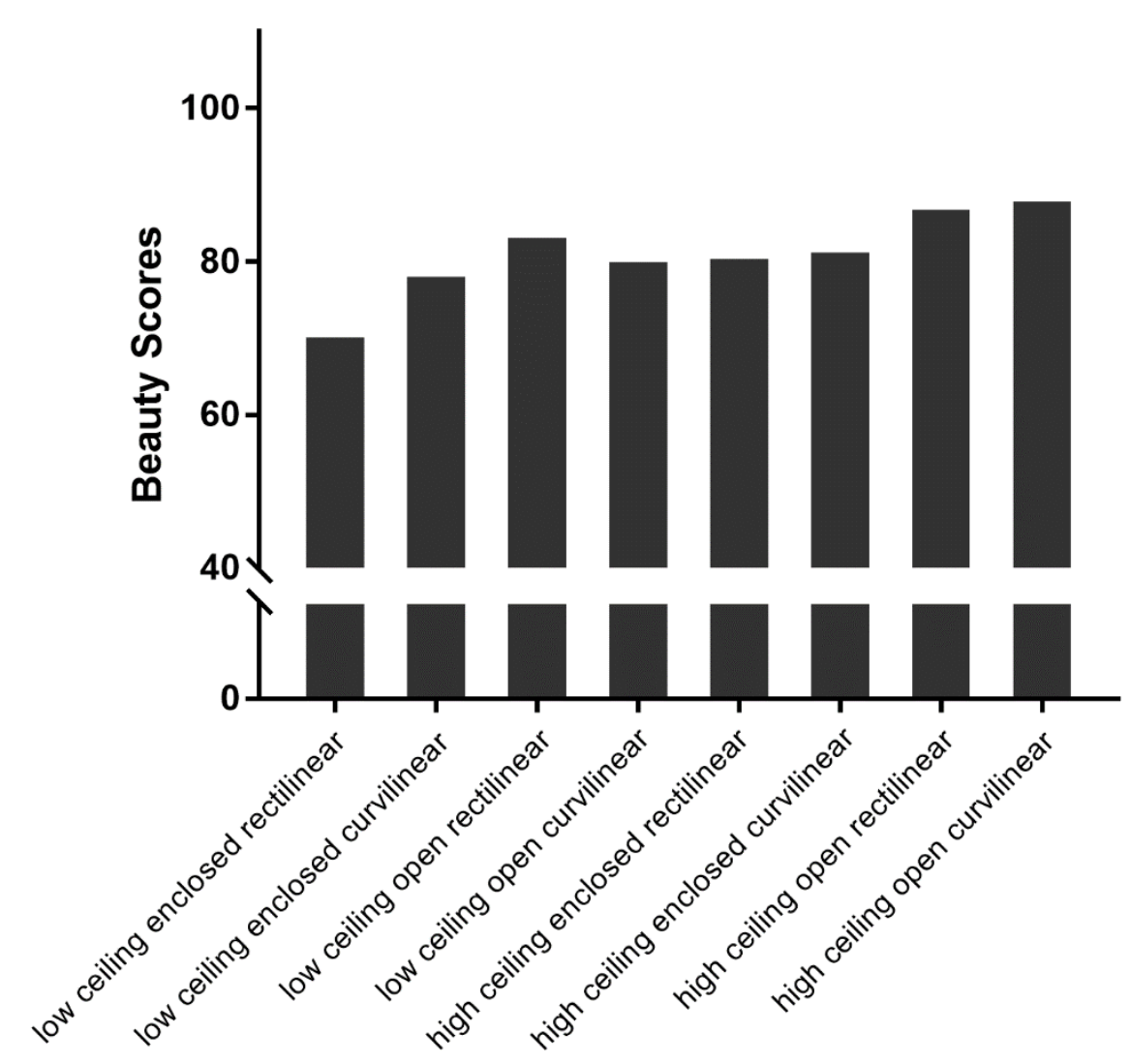


Fig 3. Beauty Rating in Different Conditions.

A. Influence of Contou

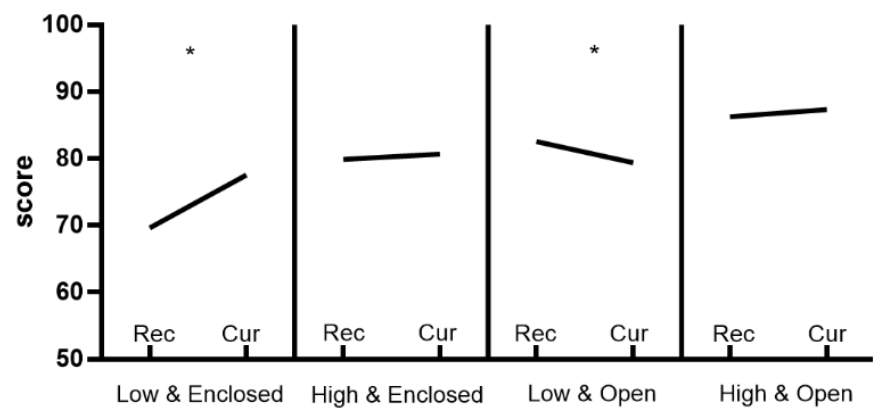

B.

Influence of Openness

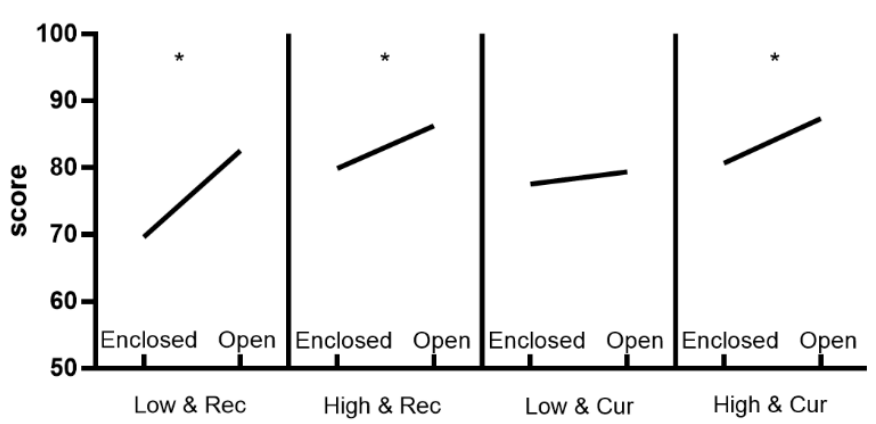

C. Influence of Ceiling height

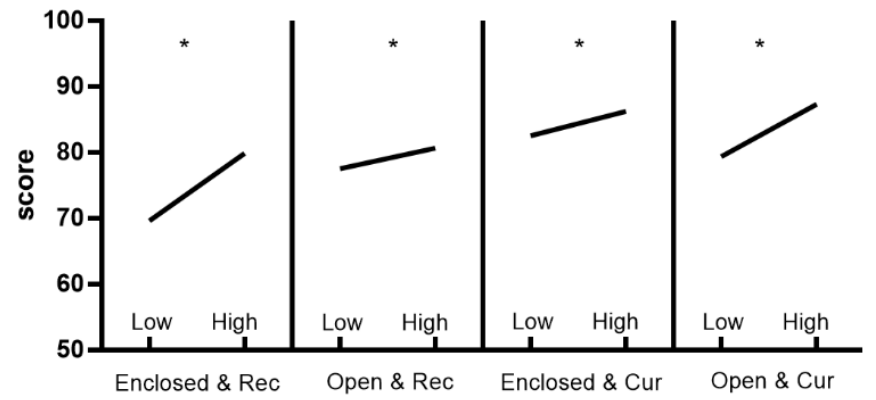


Fig 4. Scores of Pleasantness.

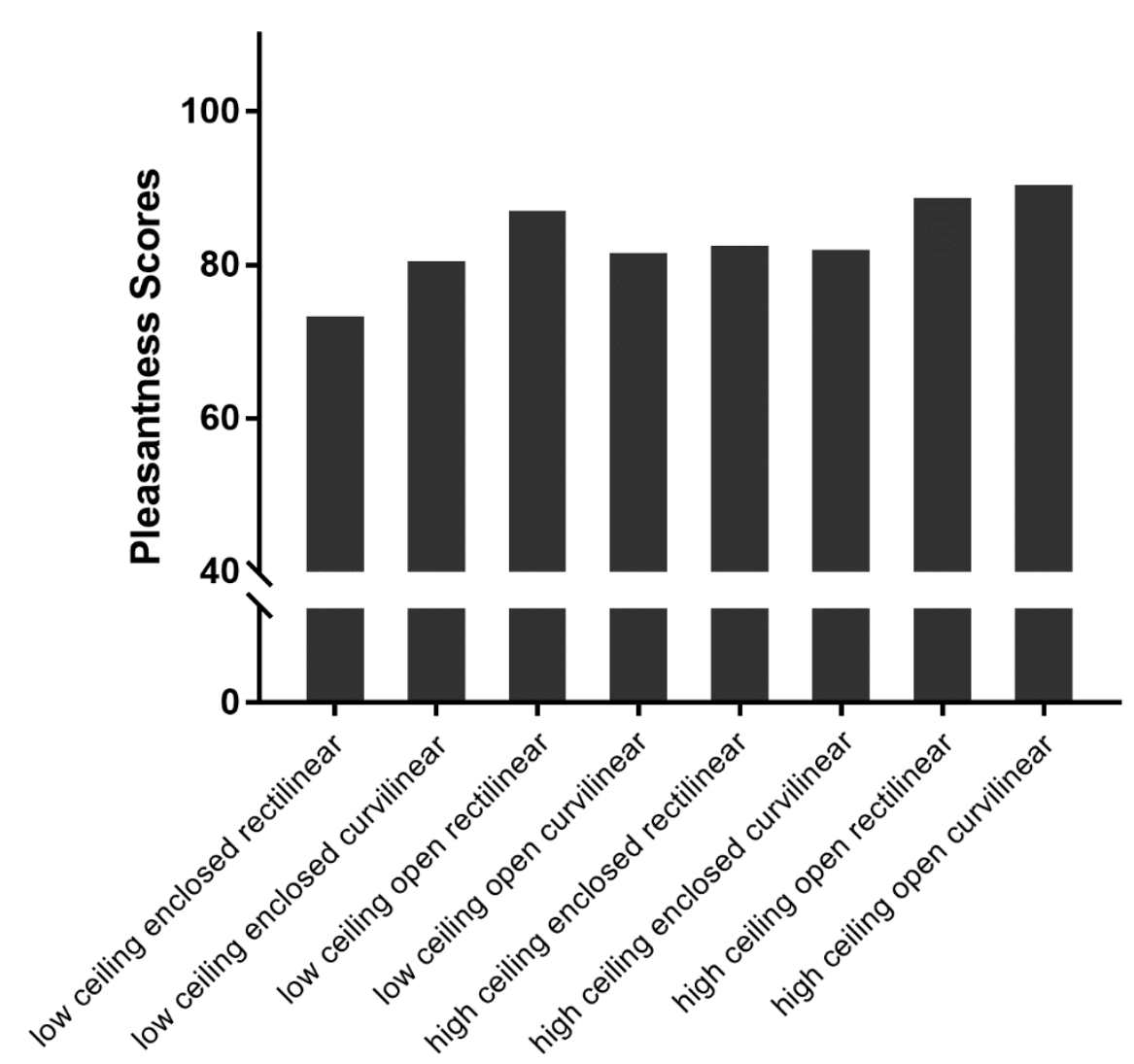


Fig 5. Pleasantness Rating in Different Conditions.

A. Influence of Contour

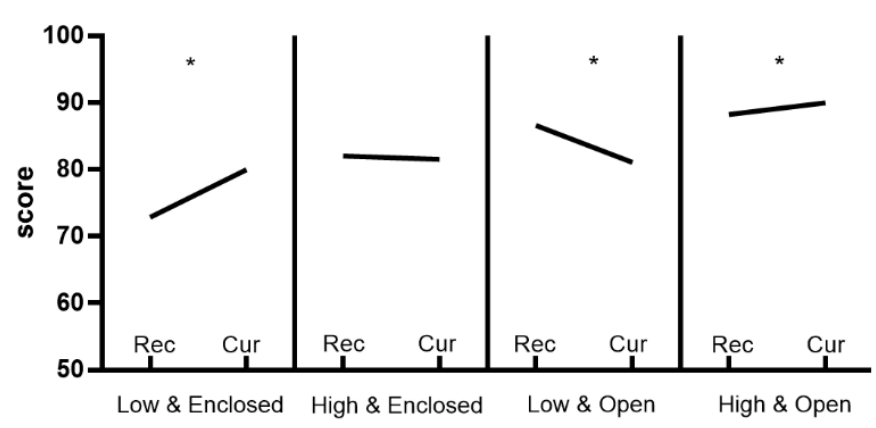

B. Influence of Openness

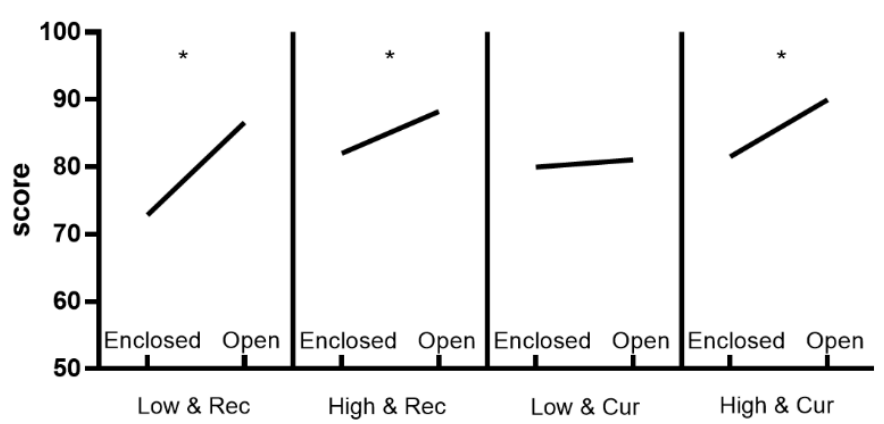

C. Influence of Ceiling height

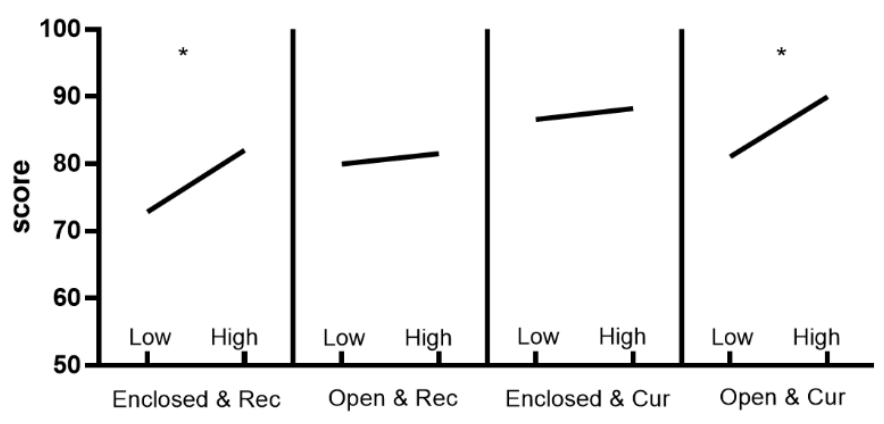


S1 Fig. the correlation
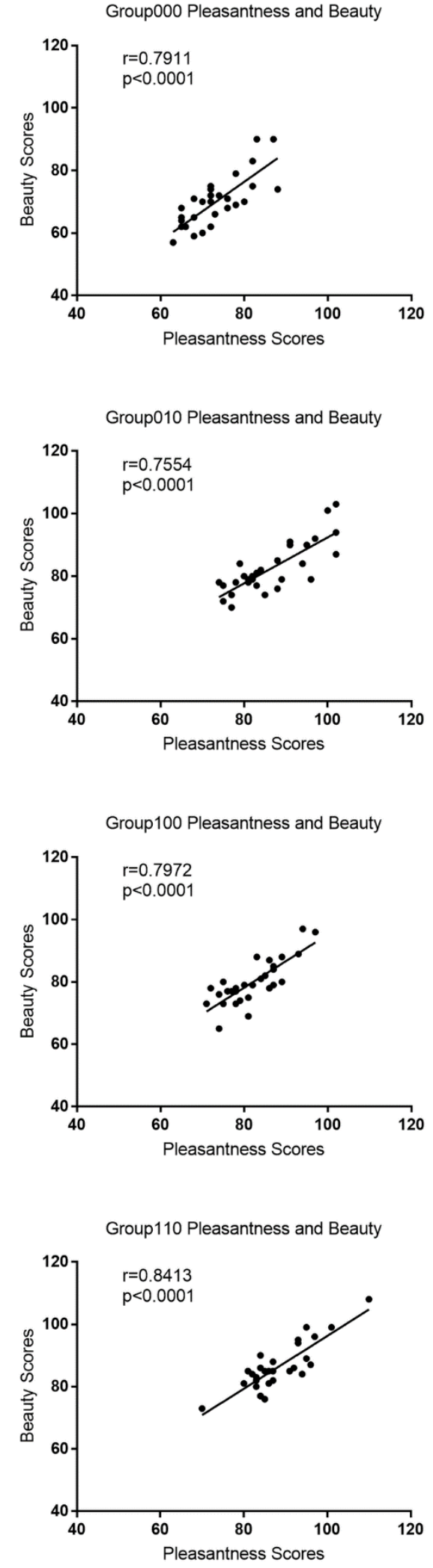
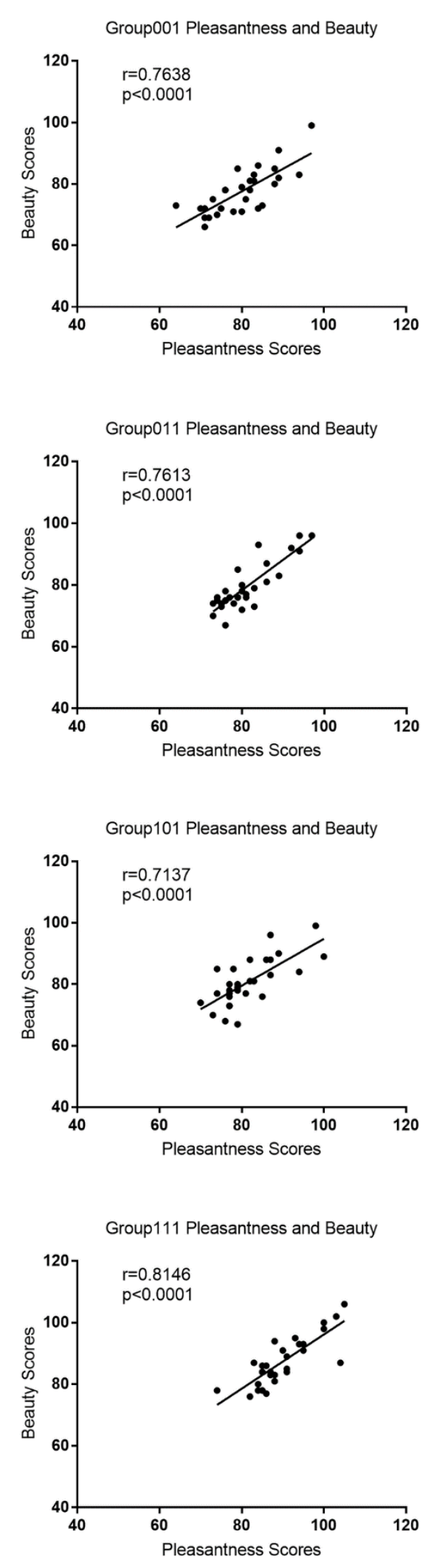